\title{
PPAR $\gamma$ Agonists Down-Regulate the Expression of Atp10c mRNA during Adipogenesis
}

\author{
Amanda L. Peretich ${ }^{1}$, Maria Cekanova ${ }^{2}$, Sarah Hurst ${ }^{1}$, Seung J. Baek ${ }^{2}$ and Madhu S. Dhar ${ }^{*}, 1$ \\ ${ }^{I}$ Departments of Large Animal Clinical Sciences and ${ }^{2}$ Pathology, College of Veterinary Medicine, University of \\ Tennessee, Knoxville, TN 37996, USA
}

\begin{abstract}
We have shown that Atp10c, a type 4 P-type ATPase, is a strong candidate affecting glucose and lipid metabolism in humans and mice. Atp10c is a putative phospholipid translocase associated with cell signaling and intracellular protein trafficking. In order to examine the biological role of Atp10c, semiquantitative reverse transcriptase polymerase chain reaction was carried out. Atp10c mRNA is expressed in 3T3-L1 cells and in primary preadipocytes and adipocytes generated from mice. Atp10c mRNA is regulated during fat cell differentiation and modulated by PPAR $\gamma$ agonists and antagonists as well as by hormonal factors (insulin and dexamethasone). Atp10c expression is regulated by both the process of adipocyte differentiation and by effectors of fat and glucose metabolism. Taken together these data along with the published phenotype of the Atp $10 \mathrm{c}$ heterozygote mice suggest that ATP10C is a newly identified protein with a possible biological role in the development of obesity and obesity-related metabolic disorders.
\end{abstract}

\section{INTRODUCTION}

Studies in yeast demonstrate that members of a novel type 4 subfamily of P-type ATPases are important in protein trafficking in the exocytic and endocytic pathways. These putative phospholipid translocases (PLTs), or flippases, presumably function to control cellular integrity and homeostasis by maintaining the lipid bilayer [1-4]. PLTs have been implicated in the translocation of phospholipids from the outer leaflet to the inner leaflet of lipid bilayers. Mutations in the sequences of mammalian flippases show their association with human disorders of intramembranous transport. For instance, mutations in human ATP8B1 are associated with Byler disease, which is a severe inherited disease of the liver. Studies in mice show that a deficiency of Atp $8 b 1$ causes a loss of phospholipid asymmetry, which subsequently impairs bile salt transport and causes cholestasis [4-7]. Another member of this subfamily, the murine gene Atp10d, has been mapped within a quantitative trait locus (QTL) associated with HDL-cholesterol levels in the C57BL/6 strain of mice [8]. Although there is consensus on the physiological function for PLTs, the molecular and cellular mechanisms by which these proteins function are poorly understood.

Atp10c is a putative PLT located on mouse chromosome 7 that has been associated with obesity, type 2 diabetes, and nonalcoholic fatty liver disease in mice. Heterozygous mice inheriting a maternal deletion of the Atp10c gene become obese and show hyperinsulinemia, glucose intolerance, and insulin resistance. Mutants show a $30-35 \%$ decrease in insulin-stimulated glucose uptake in the peripheral tissues. Gene expression profiling shows a dramatic, diet-induced loss of Atp10c expression in the soleus muscle of mutants

*Address correspondence to this author at the College of Veterinary Medicine, 2407 River Drive, Knoxville, TN 37996, USA; Tel: +1 865974 5691; Fax: +1 865974 5773; E-mail: mdhar@utk.edu along with subtle changes in adipocytes. Significant changes in the biochemical and molecular response of fat and muscle tissues in Atp10c mutants indicate an important role of ATP10C in the insulin signaling pathways that mediate glucose clearance by peripheral tissues [9-11]. We have also identified and characterized the human ortholog, ATP10C, and have mapped it to the syntenic region on chromosome 15, HSA 15 [12]. A group of neurologically affected patients with the additional phenotype of increased body mass index who carry deletions in this region has also been reported, thus suggesting an association of Atp10c/ATP10C with some forms of glucose and lipid metabolism disorders in both mice and humans [13-15]. In general, data suggest a probable interaction between PLTs and obesity and insulin resistance, clearly emphasizing the importance of investigating their exact biological role(s).

In the study of metabolic diseases, the ability to distinguish between primary and secondary effects is a major problem. Given that Atp10c heterozygote mice are obese and exhibit metabolic abnormalities, it is difficult to understand if changes in glucose and lipid metabolism directly arise from alterations in ATP10C activity or if they are due to secondary complications. Thus, the present study was initiated to define the functional importance of Atp10c in a cell. Our objective was two-fold: to establish a cell system expressing Atp10c, then to use this cell system to identify factors modulating Atp10c mRNA expression. 3T3-L1 cells, a commercially available mouse cell line [16], and purified preadipocytes and adipocytes from wild-type mouse fat tissue were used.

\section{MATERIALS AND METHODS}

\subsection{Materials}

Cell culture reagents and oligonucleotides were obtained from Fisher Scientific (Fairlawn, NJ). Insulin, isobutyl- 
methylxanthine, isoproterenol, dexamethasone, all-trans retinoic acid, and fetal bovine serum were purchased from Sigma (St. Louis, MO). Thiazolidinediones MCC555 and troglitazone (TGZ) and the PPAR $\gamma$ inhibitor GW9662 were obtained from Cayman (Ann Arbor, MI).

\subsection{Cell Culture}

3T3-L1 preadipocytes obtained from ATCC (Manassas, VA) were grown at $37^{\circ} \mathrm{C}$ in $5 \% \mathrm{CO}_{2}$ in Dulbecco's modified Eagle's medium containing $10 \%$ fetal bovine serum and $1 \%$ penicillin/streptomycin (growth medium). At 70-80\% confluency, adipocyte differentiation was induced by incubating the cells in growth medium supplemented with $0.5 \mathrm{mM}$ isobutylmethylxanthine, $1 \mu \mathrm{M}$ dexamethasone, and $1 \mu \mathrm{g} / \mathrm{ml}$ insulin (differentiation medium) for two days. After 2 days, cells were maintained in growth medium supplemented with $1 \mu \mathrm{g} / \mathrm{ml}$ insulin (maintenance medium) and grown for 7-10 days; the medium was changed every two to three days. Cells were fully differentiated $(>80 \%)$ by day $8-10$ as assessed by Oil Red $\mathrm{O}$ staining and reverse transcriptase PCR (RT-PCR); these are 3T3-L1 adipocytes. Day 0 represents the day on which the differentiation media is replaced by maintenance media.

Confluent 3T3-L1 preadipocytes or adipocytes were incubated for 24 hours in serum-free medium before various effectors were added as described in the figure legends. PPAR $\gamma$ agonists and antagonists were replenished every time the media was changed.

Primary preadipocytes and adipocytes were isolated from adipose tissue of 4-6 month old adult female wild-type mice from ongoing crosses of Atp 10c heterozygotes, using Rodbell's procedure with modifications [17, 18]. Approximately $2 \mathrm{~g}$ of tissue was collected, minced into small pieces, and digested with collagenase $(2 \mathrm{~g} / \mathrm{L})$ (type I, Worthington Biochemical Corp., Lakewood, $\mathrm{NJ}$ ) at $37^{\circ} \mathrm{C}$ for $25 \mathrm{~min}$ in modified Krebs-Ringer bicarbonate albumin buffer $\mathrm{pH} 7.4$, containing $1 \mathrm{~g} / \mathrm{L}$ BSA and $4.4 \mathrm{mM}$ glucose. Digested fat tissue was filtered through a $100 \mu \mathrm{m}$ nylon mesh and primary adipocytes were isolated by a low speed centrifugation at $800 \mathrm{rpm}$ for $10 \mathrm{~min}$. These adipocytes were washed once with Hank's balanced salt solution and collected. The stromal vascular fraction rich in primary preadipocytes was then pelleted by centrifugation at $1500 \mathrm{rpm}$ for $15 \mathrm{~min}$. Resulting stromal vascular fraction cell pellets were treated with red blood cell lysis buffer (eBioscience, San Diego, CA) for $5 \mathrm{~min}$ at room temperature, rinsed with phosphate buffered saline supplemented with 1\% BSA, and centrifuged at $800 \mathrm{rpm}$ for $10 \mathrm{~min}$. Stromal vascular fraction cells were seeded in 6-well cell culture plates at a density of about $3 \times 10^{5}$ cells/well.

At $70-80 \%$ confluency, primary preadipocytes in the stromal vascular fraction cells were induced to differentiate in growth medium supplemented with $10 \mu \mathrm{g} / \mathrm{ml}$ insulin (high insulin medium). Differentiation was complete in 8-10 days as assessed by Oil Red O staining and RT-PCR. These cells are the primary adipocytes obtained from primary preadipocytes differentiated in culture.

All animal procedures were in accordance with the University of Tennessee IACUC (protocol \#1309-1206).

\subsection{Oil Red O Staining}

Adipocyte differentiation was assessed using a modified procedure of Oil Red O staining [19]. Cells were washed with Hank's balanced salt solution and fixed with $1 \%$ paraformaldehyde for $5 \mathrm{~min}$. Cells were rinsed with deionized water followed by the addition of two aliquots of $85 \%$ propylene glycol for 5 min each. Oil Red O stain was added $(0.7 \% \mathrm{w} / \mathrm{v}$ in $85 \%$ propylene glycol) and cells were gently swirled for $20 \mathrm{~min}$ at room temperature. Following a final wash with $85 \%$ propylene glycol for $3 \mathrm{~min}$, cells were counterstained with hematoxylin. They were visualized and photographed using a Zeiss Invertoskop 40C microscope under 10X magnification and captured by a Canon Powershot A620 digital camera.

\subsection{Semiquantitative RT-PCR}

These procedures are as previously described with a few modifications [10]. Total RNA was extracted from the harvested cells using RNeasy Mini RNA kit (Qiagen Inc. Valencia, CA) according to the manufacturer's instructions. About $1 \times 10^{6}$ cells yield $30-50 \mu \mathrm{g}$ of total RNA. First strand cDNA was synthesized using the iScript cDNA synthesis kit (Bio-Rad, Hercules, CA). Semiquantitative RT-PCR was performed for Atp10c (F-5'CCTGTGCTCTTCATTCTG GC3', R-5'CACTGCAGCTGTGAATCTGT3'), resis-tin (F-5'ACTGAGTTGTGTCCTGCTAAG3', R-5'CCACGCT CACTTCCCCGACATC $\left.3^{\prime}\right)$, and PPAR $\gamma$ (F-5'GGTGAA ACTCTGGGAGATTC3', R-5'CAACCATTGGGTCAGCT CTT3') mRNAs using GoTaq ${ }^{\circledR}$ Green Master Mix (Promega Inc. Madison, WI) with $\beta$-actin (F-5'ATGGGTCAGAAGG ACTCCTA3', R-5'CAACATAGCACAGCTTCTCT3') as an internal control. RT-PCR was performed under the following conditions: $5 \mathrm{~min}$ at $94^{\circ} \mathrm{C}$ followed by 30 cycles of denaturation at $94^{\circ} \mathrm{C}$ for $30 \mathrm{~s}$, annealing at $54^{\circ} \mathrm{C}$ for $30 \mathrm{~s}$, and extension at $72^{\circ} \mathrm{C}$ for $30 \mathrm{~s}$, and finishing with $5 \mathrm{~min}$ at $72^{\circ} \mathrm{C}$. RT-PCR products were analyzed on $1 \%$ agarose gels containing $1 \mu \mathrm{g} / \mu \mathrm{l}$ ethidium bromide and viewed under ultraviolet light.

\subsection{Data Analysis}

RT-PCR products on the agarose gels were quantitated using Scion Image (http://www.scioncorp.com). Results are expressed as the ratio of the target gene expression to that of $\beta$-actin and are shown as means \pm SD. Mean comparisons were tested by two-tailed unpaired Student's $t$-test with $P$ values $<0.01$ considered highly significant and $<0.05$ considered significant.

\section{RESULTS}

Using RT-PCR, an 886 bp product was detected in both the undifferentiated and differentiated 3T3-L1 cells. This product has been demonstrated and confirmed to be a part of Atp10c cDNA [11]. Quantitative analysis showed that Atp10c is 2-fold down-regulated in 3T3-L1 adipocytes after differentiation (Fig. 1). Atp10c mRNA is also expressed in purified preadipocytes and adipocytes from mouse adipose tissue and in primary preadipocytes differentiated in culture (Fig. 2). Compared to primary preadipocytes, Atp 10c mRNA is significantly down-regulated in primary adipocytes, again confirming the regulation of Atp10c expression during 


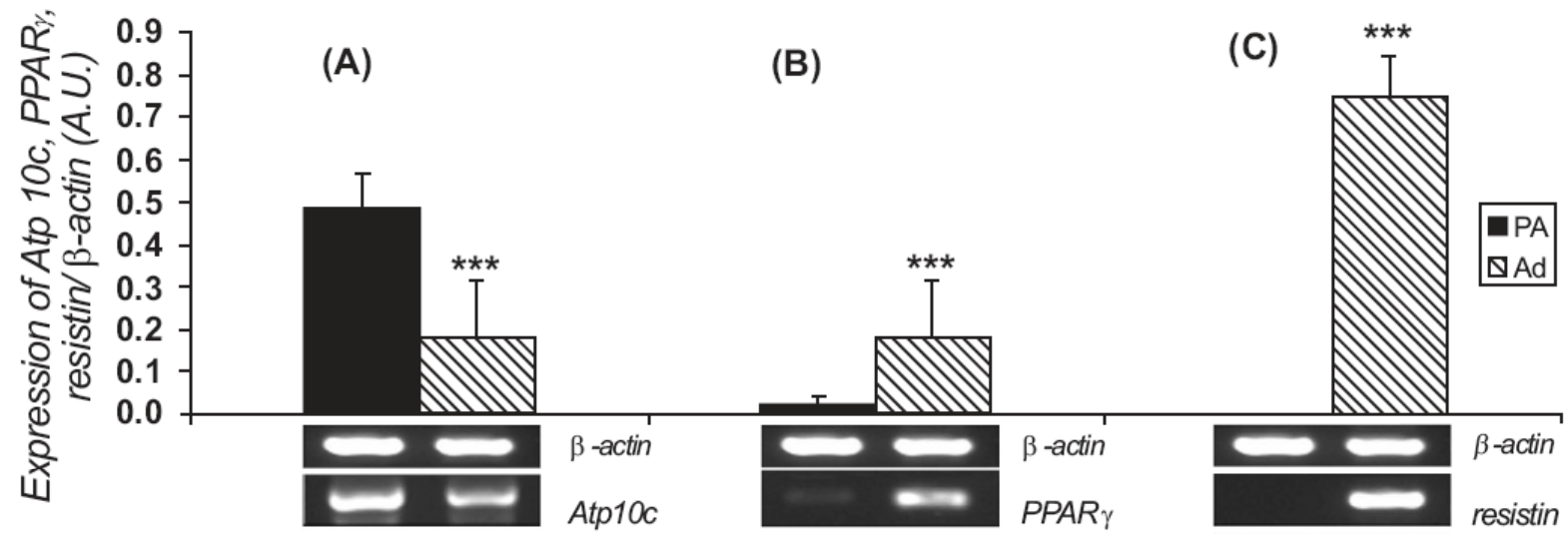

Fig. (1). Atp10c mRNA is expressed in 3T3-L1 cells. Atp10c (A), resistin (B), and PPAR $\gamma(\mathbf{C})$ expression was examined by RT-PCR in 3T3L1 preadipocytes (PA) and adipocytes (Ad); $\beta$-actin served as an internal control. Expression of Atp10c, PPAR $\gamma$ and resistin mRNAs is denoted as arbitrary units (A.U.) and represented as normalized to $\beta$-actin. Data from four independent experiments are shown. $* * * P<0.001$. Lack of PCR products due to low levels of expression are denoted as not detected (ND).

adipogenesis. As expected, PPAR $\gamma$ (PCR product of size $268 \mathrm{bp}$ ) and resistin (PCR product of size $386 \mathrm{bp}$ ) are upregulated, serving as internal positive controls (Fig. 2A).

Adipose tissue is comprised of several types of cells such as fibroblasts, non-differentiated mesenchymal cells, preadipocytes, and adipocytes. Adipocytes develop from fibroblast-like preadipocytes within the stromal vascular fraction
$[20,21]$. Hence, to ensure that stromal vascular fraction cells in our preparations were comprised mainly of preadipocytes and that Atp10c expression was specific to the preadipocyte fraction, we differentiated the stromal vascular fraction cells to adipocytes, and then measured Atp10c expression. Interestingly, Atp10c expression was about 2-fold upregulated whereas, $P P A R \gamma$ and resistin expressions were

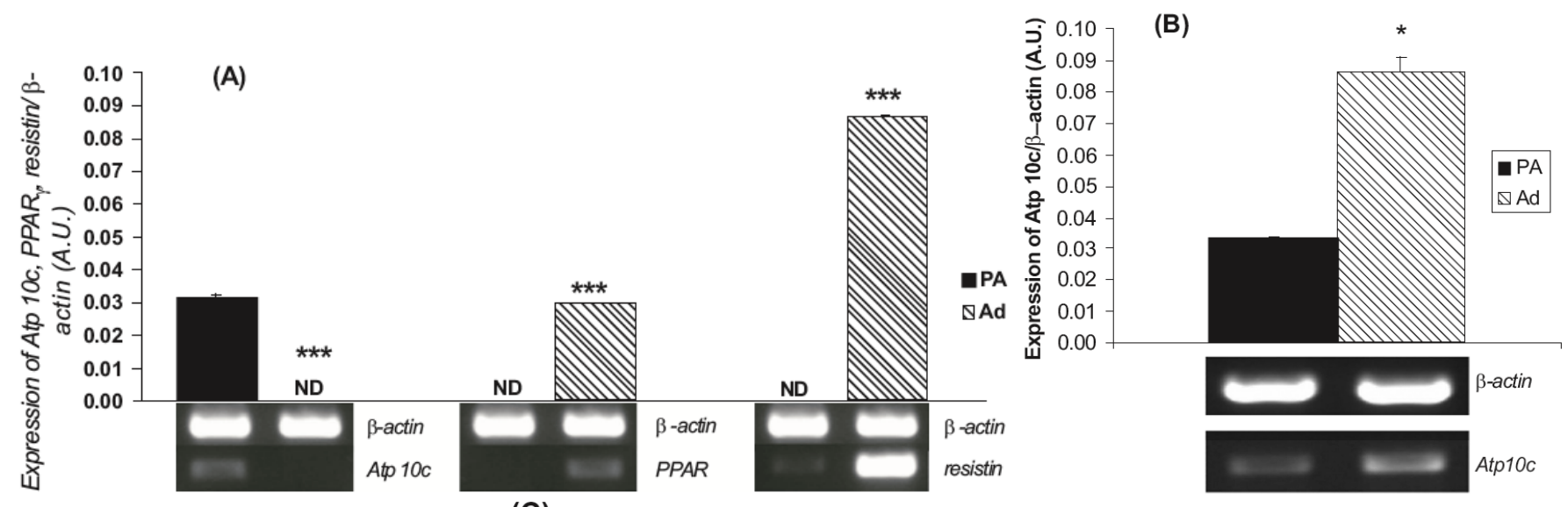

(C) PA

Ad

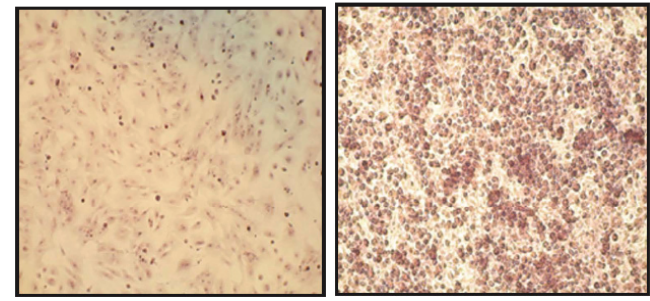

Fig. (2). Atp10c mRNA is expressed in primary preadipocytes and adipocytes. RT-PCR analysis was used to determine Atp10c, resistin, and $P P A R \gamma$ expression in primary preadipocytes (PA) and adipocytes (Ad) purified from mouse adipose tissue (A); $\beta$-actin served as an internal control. Expressions are denoted as arbitrary units (A.U.) and represented as normalized to $\beta$-actin. Data from four independent experiments are shown. ${ }^{* * *} P<0.001$. Lack of PCR products due to low levels of expression are denoted as not detected (ND).

RT-PCR analysis was used to determine Atp10c expression in primary preadipocytes (PA) and adipocytes (Ad) harvested from culture (B); $\beta$-actin served as an internal control. Expression is denoted as arbitrary units (A.U.) and represented as normalized to $\beta$-actin. Data from four independent experiments are shown. ${ }^{* * *} P<0.001$.

Oil Red O staining (C) was used to monitor and confirm differentiation of primary preadipocytes (PA) to adipocytes (Ad) in culture. A representative image from four independent experiments is shown. 

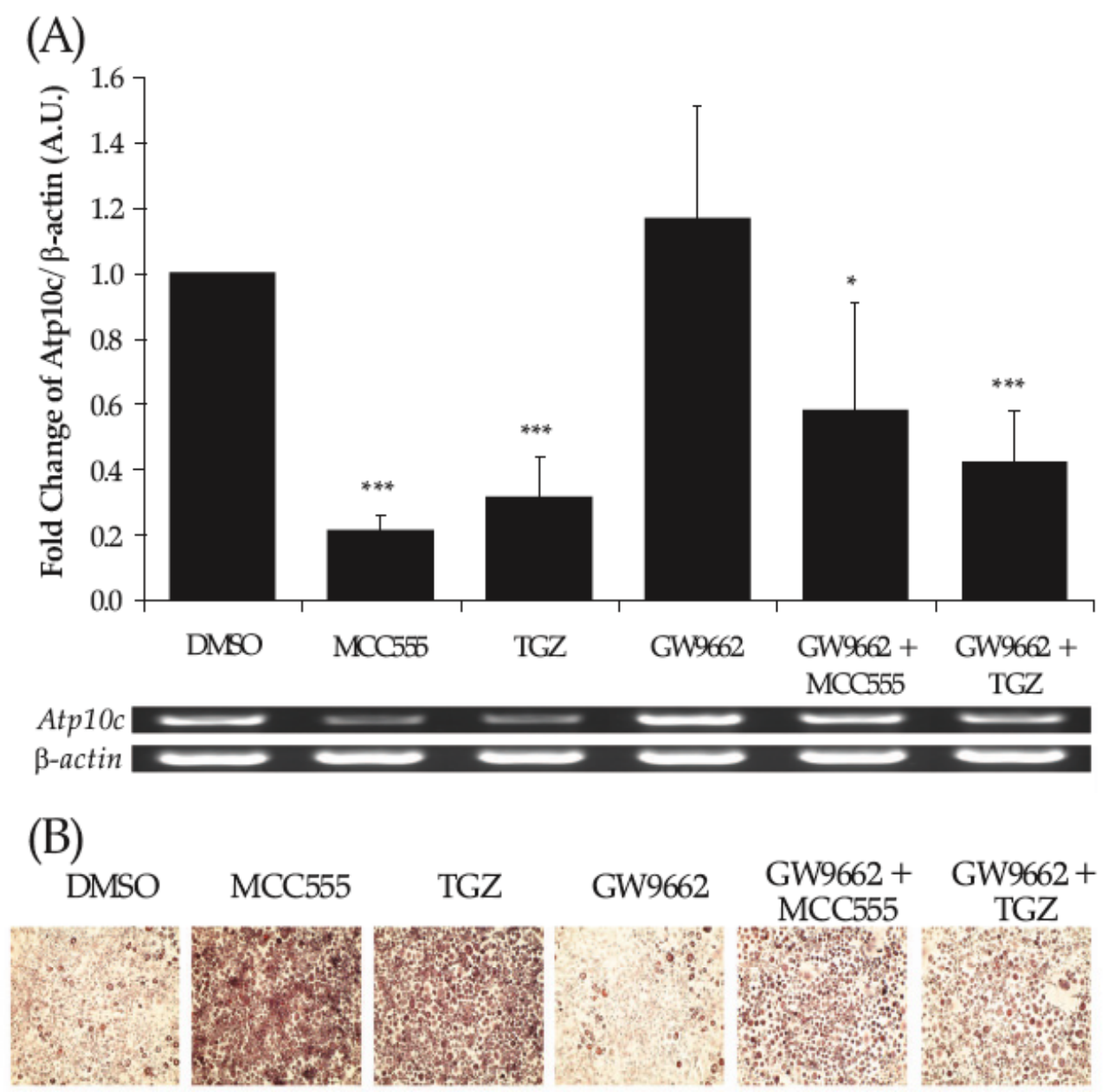

Fig. (3). Atp10c mRNA expression is regulated by PPAR $\gamma$ agonists and antagonists. RT-PCR analysis shows Atp10c expression in 3T3-L1 adipocytes harvested between days 8-10 of differentiation. MCC555, troglitazone (TGZ), and GW9662 were added to the media throughout the differentiation process and replenished whenever the media was changed. Expression of Atp10c mRNA in adipocytes treated with $10 \mu \mathrm{M}$ DMSO (vehicle), $10 \mu \mathrm{M}$ MCC555, 10 $\mu \mathrm{M}$ TGZ, and $10 \mu \mathrm{M}$ GW9662 is shown (A), with $\beta$-actin serving as an internal control. The expression of Atp10c is denoted as arbitrary units (A.U.) and represented as the fold change normalized to $\beta$-actin. Oil Red O staining (B) was used to monitor morphological changes in adipocytes in the presence of PPAR $\gamma$ agonists and antagonists. Data from six independent experiments are shown. $* P<0.05, * * * P<0.001$.

significantly down-regulated (Fig. 2B). PPAR $\gamma$ and resistin mRNA expressions were very low in primary preadipocytes and after differentiation no PCR product was detected in primary adipocytes. As described below, we believe that this increase in Atp10c expression and the corresponding decrease in PPARy and resistin expressions in primary adipocytes are due to high insulin present in the differentiation media used for primary cultures. Oil Red $\mathrm{O}$ staining was used to monitor differentiation in culture and to confirm that the harvested cells showed $>80 \%$ differentiation (Fig. 2C).

The process of adipocyte differentiation is associated with a large number of cis -and trans-acting factors [22-24]. The regulation of different genes at distinct times after the exposure of cells to differentiation conditions suggests the existence of a regulatory hierarchy or cascade of events $[25$, 26]. PPAR $\gamma$ is considered to be the master regulator of adipogenesis, controlling the expression of adipocyte genes. Therefore, we investigated the effect of PPAR $\gamma$ agonists on Atp10c expression to assess whether Atp10c expression is also under PPAR $\gamma$ control. Two anti-diabetic drugs that acti- vate PPAR $\gamma$ and thus promote adipogenesis, MCC555 and TGZ, were used [27, 28]. 3T3-L1 adipocytes were treated with these reagents as described in materials and methods and in figure legends. As demonstrated by RT-PCR and Oil Red O staining, both MCC555 and TGZ promoted adipogenesis and consequently reduced Atp10c expression by 5 -fold and 3-fold respectively (Fig. 3). This significant decrease in adipocytes further confirms the pattern of Atp10c expression in differentiated cells.

To clarify and confirm that the decrease in Atp10c is indeed due to the process of adipogenesis controlled by PPAR $\gamma$, Atp 10c expression was also assessed in cells treated with a PPAR $\gamma$ antagonist, GW9662 [29]. Atp10c expression in adipocytes treated with GW9662 alone did not decrease significantly and was similar to that in control cells treated with the vehicle DMSO. However, when GW9662 was added to the cells along with MCC555 or TGZ, there was only about a 2 -fold decrease in Atp10c expression. This suggests that GW9662 inhibited adipogenesis and rescued some of the decrease in Atp10c expression due to MCC555 or TGZ alone. Similarly, Atp10c expression did not decrease 
(A)

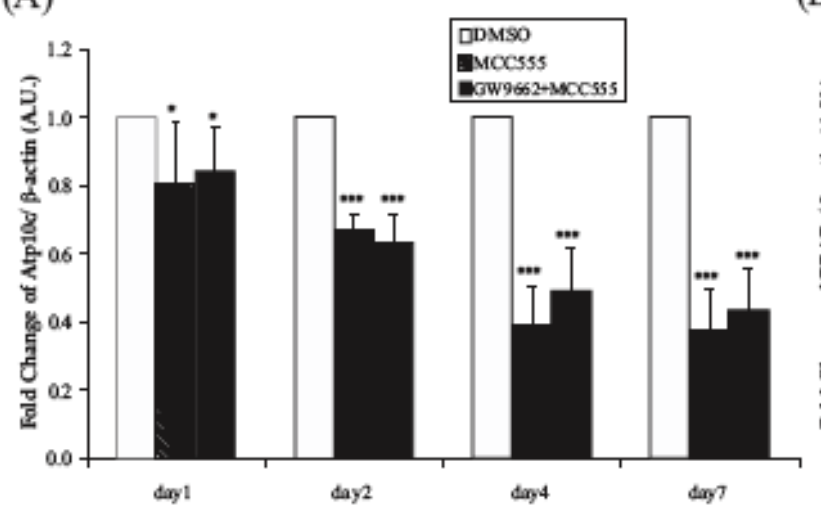

(B)

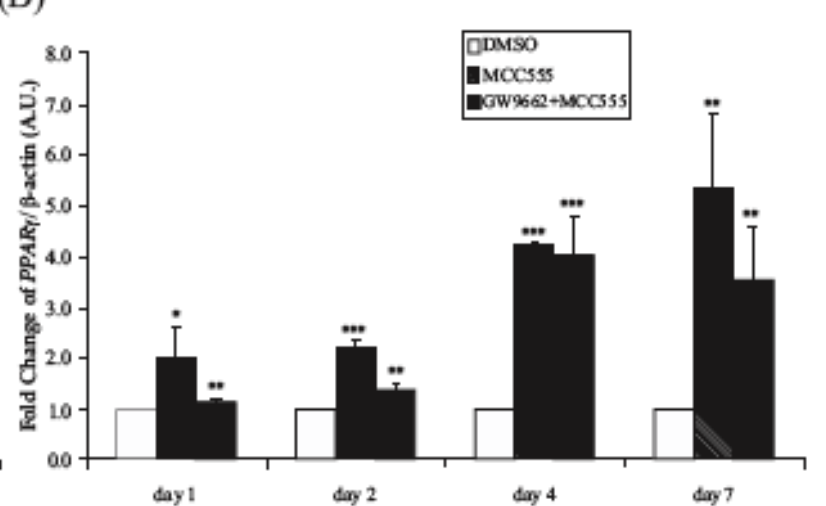

(C)

day 1

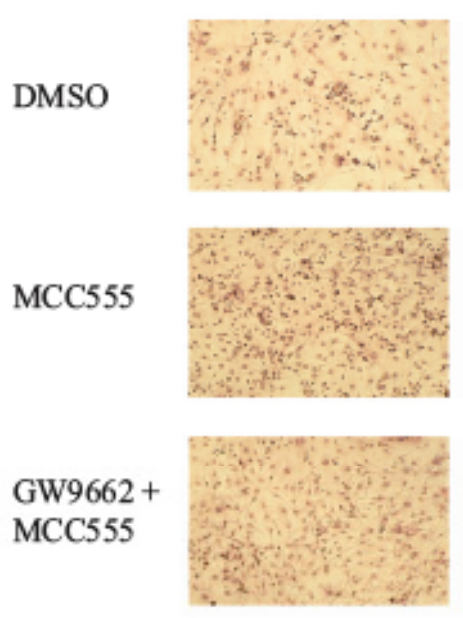

day 2
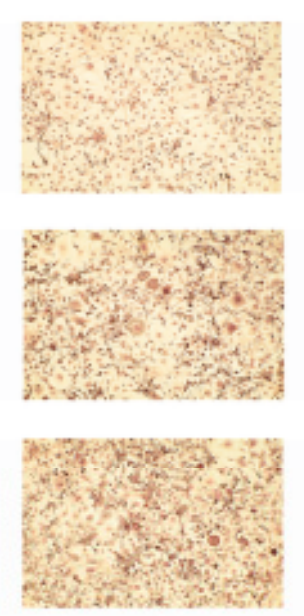

day 4
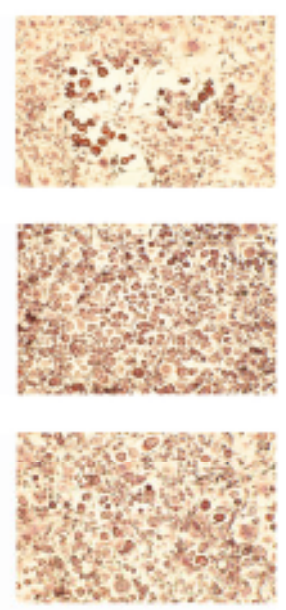

day 7
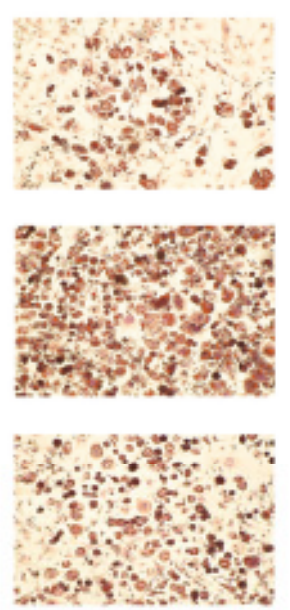

Fig. (4). Atp $10 c$ and PPAR $\gamma$ mRNAs are oppositely regulated in 3T3-L1 cells during adipogenesis. RT-PCR analysis was used to determine Atp10c (A) and PPAR $\gamma(\mathbf{B})$ mRNA expression in 3T3-L1 cells at days 1, 2, 4, and 7 post-induction; $\beta$-actin served as an internal control. Cells were treated throughout differentiation with DMSO, MCC555, or a combination of GW9662 and MCC555. Expression of Atp10c and PPAR $\gamma \mathrm{mRNA}$ is denoted as arbitrary units (A.U.) and represented as the fold change normalized to $\beta$-actin and compared to day 1 DMSO. Oil Red $\mathrm{O}$ staining $(\mathbf{C})$ was used to assess the corresponding morphological changes. Data from five independent experiments are shown. $* P<0.05, * * P<0.005, * * * P<0.001$.

following treatment of adipocytes with $1 \mu \mathrm{M}$ all-trans retinoic acid (data not shown). $1 \mu \mathrm{M}$ all-trans retinoic acid also inhibits adipocyte differentiation thus, confirming that Atp10c is highly expressed in undifferentiated cells and negatively regulated during adipocyte differentiation.

To gain further insight into the transcriptional control of Atp10c during adipogenesis Atp10c expression was compared to that of PPAR $\gamma$ in cells treated with MCC555 or a combination of MCC555 and GW9662 and harvested at days 1, 2, 4, and 7 post-induction (Fig. 4). High levels of Atp10c were expressed at day 1 that gradually decreased through days 2-7 (Fig. 4A). When $>80 \%$ of preadipocytes were differentiated into adipocytes at day 7, Atp 10c expression was 2fold down-regulated, supporting our observations described in Fig. (1). As expected, PPAR $\gamma$ expression increased significantly through days $1-7$ (Fig. 4B). Oil Red O staining was carried out to monitor the differentiation process (Fig. 4C).

Since Atp10c was expressed and levels were modulated during the differentiation of 3T3-L1 preadipocytes to adipocytes, we next investigated the regulation of Atp10c
mRNA expression by effector molecules of glucose and fat metabolism in undifferentiated and differentiated cells. This was examined by incubating cells with $100 \mathrm{nM}$ insulin, 100 $\mathrm{nM}$ dexamethasone, or $100 \mathrm{nM}$ isoproterenol for 24 hours. Atp10c mRNA increased 4-fold or 6-fold, respectively, when insulin or dexamethasone was added to 3T3-L1 adipocytes; there was no significant change in preadipocytes. As expected, resistin and PPAR $\gamma$ mRNAs decreased following treatment with insulin or dexamethasone (Fig. 5). Based on these results, we believe that the up-regulation of $A t p 10 \mathrm{c}$ and the down regulation of PPAR $\gamma$ and resistin observed in primary adipocytes differentiated in culture (Fig. 2B) are due to the high insulin used in the differentiation media.

Atp10c mRNA expression slightly decreased after incubating the cells with $100 \mathrm{nM}$ isoproterenol, but this was not statistically significant (data not shown).

\section{DISCUSSION}

Protein trafficking and intracellular signaling processes are important in controlling cellular homeostasis. Defects in 


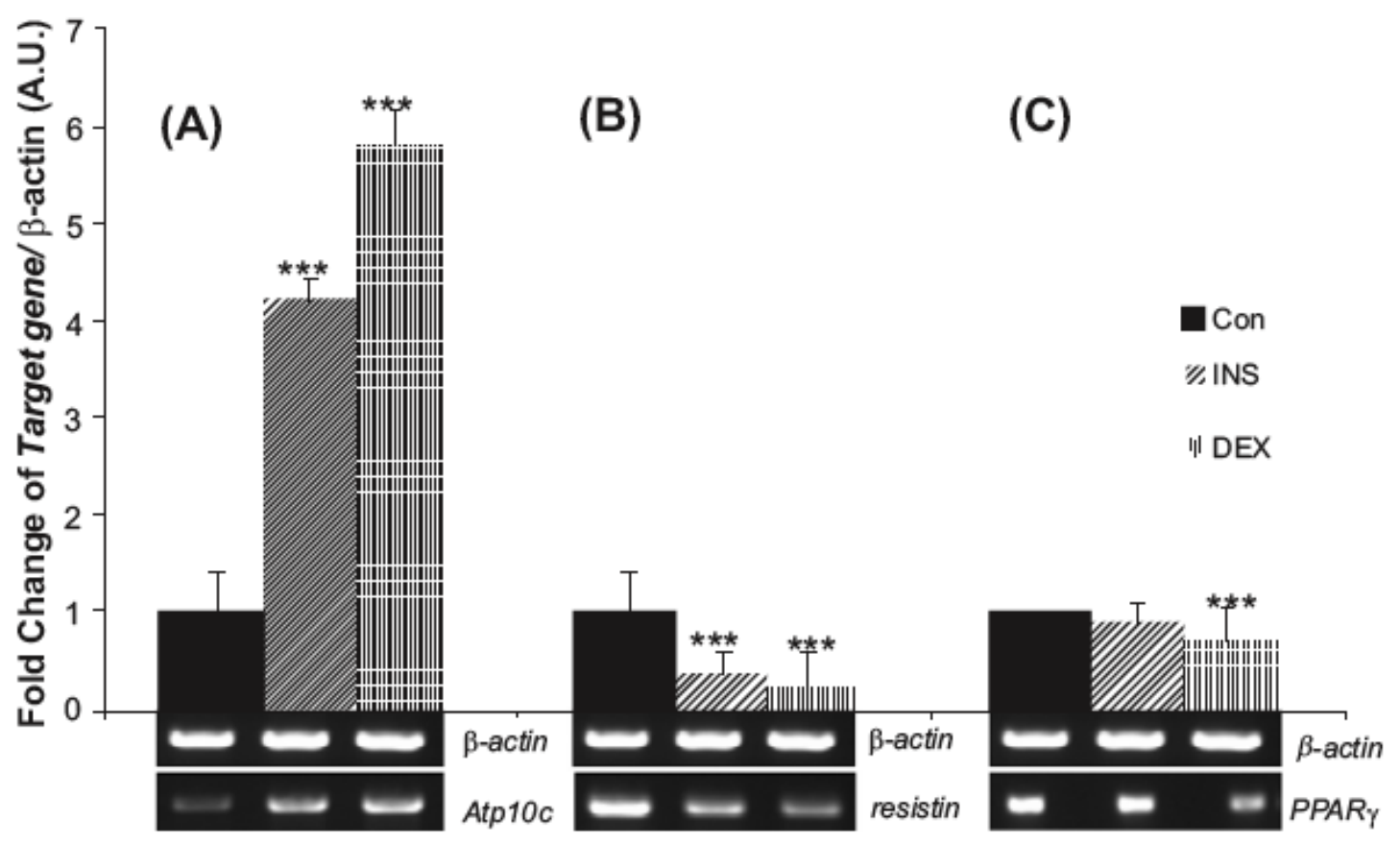

Fig. (5). Atp10c mRNA expression is regulated conversely to that of PPAR $\gamma$ and resistin mRNAs in 3T3-L1 adipocytes following treatment with hormonal factors. RT-PCR analysis was used to determine $A t p 10 c(\mathbf{A}), \operatorname{PPAR} \gamma(\mathbf{B})$ and resistin $(\mathbf{C})$ mRNA expressions in untreated 3T3-L1 adipocytes (CON), and following treatment with $100 \mathrm{nM}$ insulin (INS) or $100 \mathrm{nM}$ dexamethasone (DEX) for 24 hours. $\beta$-actin is used as an internal control. Expression is denoted as arbitrary units (A.U.) and represented as the fold change normalized to $\beta$-actin. Data from four independent experiments are shown. $* * * P<0.001$.

these processes can lead to changes in cellular function resulting in serious pathological conditions like obesity and type 2 diabetes [30,31]. Although numerous target genes and their cognate protein factors have been identified and their roles characterized, our understanding of these processes is still limited. It is probable that many components of the system are still unidentified. Hence, novel factors are continually being identified and strategies to further our understanding of mechanisms underlying the above processes are being developed.

Type 4 P-type ATPases belong to a newly identified subfamily of PLTs in eukaryotes. They are suggested to maintain the asymmetrical nature of the lipid bilayer and are proposed to be important in protein trafficking $[1,3,5]$. These ATPases are thought to catalyze the transbilayer transport of amphipathic molecules such as phospholipids, rather than being pumps for the transfer of cations [32-35]. Based on published reports from our laboratory, it is reasonable to suggest that Atp10c/ATP10C, one member of this subfamily, may play an important role in obesity of multiple etiologies in mice and humans.

Mice heterozygous for Atp10c, a putative PLT, represent a diet-induced genetic model of obesity associated with insulin resistance. Prior studies suggest that misexpression of Atp10c promotes obesity by modulating glucose and lipid metabolism $[9,10]$. To gain mechanistic insight into the link between the translocase activities of PLTs and metabolic diseases, in the current report we have successfully established cell culture systems which express Atp $10 \mathrm{c}$ and thus, will be used to study its functional role(s) in insulin signaling and adipogenesis.
We show for the first time here that Atp10c mRNA is expressed in the undifferentiated and differentiated adipocytes both in vitro and ex vivo. We also demonstrate changes in Atp 10c expression during adipose cell differentiation. Finally, we report that Atp10c expression in 3T3-L1 adipocytes is significantly altered by peroxisome proliferatoractivated receptor- $\gamma$ (PPAR $\gamma$ ) agonists and antagonists as well as by the hormonal factors insulin and dexamethasone. A solid understanding of Atp $10 \mathrm{c}$ mRNA will now assist in the future studies to understand the regulation of ATP10C protein.

Adipocyte differentiation involves a temporally regulated set of gene expression events, and understanding the underlying transcriptional networks is of fundamental importance to adipocyte biology. PPAR $\gamma$, a member of the nuclearreceptor superfamily, is considered as a master regulator of adipogenesis. It is both necessary and sufficient for adipogenesis. Numerous pro- and anti-adipogenic factors work via PPAR $\gamma$ to activate or repress adipocyte differentiation [36, 37]. Almost every important cellular signaling pathway exerts a positive or negative effect on adipocyte development via factors that are still poorly understood. As a result, the identification and characterization of novel factors affecting these processes is ongoing. 3T3-L1 cells are being used extensively as an in vitro model and primary cultures derived from mouse adipose tissue as an ex vivo model to study the cellular and molecular aspects of adipocyte differentiation.

Interestingly, our data also shows that Atp10c is a novel factor negatively regulated during adipogenesis. We have shown that Atp10c mRNA is significantly down-regulated by the process of adipocyte differentiation and that these changes are not due to any experimental manipulation of the 
cells in culture. The differential expression of Atp $10 c$ in preadipocytes and adipocytes is an example of cell typespecific expression similar to that of pref-1, where the gene is active in preadipocytes and is repressed in adipocytes [38]. Atp10c may be an important component of preadipocytes, functioning to maintain a preadipose state or to prepare the cells for the process of differentiation. The exact role of Atp10c in adipogenesis is not known at this time; however, our data clearly establishes a pattern completely opposite to that of PPAR $\gamma$ and some other well-characterized obesity genes like resistin. Our data suggests a regulatory control of Atp10c transcription during adipocyte development and lends further support to the use of the 3T3-L1 cell line. Specific experiments to study translational control and the effect of ATP10C - silencing or - overexpression on the other obesity/diabetes genes can now be accomplished.

PPAR $\gamma$ agonists, such as thiazolidinediones, promote adipocyte differentiation and have insulin-sensitizing effects in animals and diabetic patients [25-27]. Conversely, the PPAR $\gamma$-specific antagonist GW9662 has been shown to inhibit adipogenesis and has been successfully used to study the regulation of gene expression during 3T3-L1 adipocyte differentiation [29]. Similarly, retinoic acid prevents and antagonizes PPAR $\gamma$ gene expression and is shown to be an inhibitor of adipocyte differentiation [39]. Thus, down-regulation of Atp10c expression by the PPAR $\gamma$ agonists MCC555 and TGZ along with restoration of Atp10c expression by PPAR $\gamma$ antagonists GW9662 and retinoic acid, suggest a possible relationship between PPAR $\gamma$-mediated adipogenesis and $A t p 10 c$ transcription. Whether a similar association exists between adipogenesis and ATP10C translation is to be determined.

Finally, our results also suggest transcriptional control of Atp10c expression by the hormonal factors insulin and dexamethasone during differentiation. Insulin is already known to regulate a number of mRNAs coding for obesity and diabetes genes $[39,40]$. Hence, in this study, we prove that insulin regulates yet another novel gene, Atp10c, again suggesting it to be important in insulin signaling processes. Dexamethasone induces insulin resistance and stimulates Atp10c production, which is similar to its effect on $o b$ gene expression and leptin production [41]. In contrast, lack of a significant effect by isoproterenol suggests that Atp10c expression is not controlled by adrenoceptors in 3T3-L1 adipocytes [42].

In summary, the results of this study strengthen our earlier hypotheses that $A t p 10 c$ has a putative role in glucose and lipid metabolism. However, the exact mechanism is still unknown. Even though we have not yet proved our hypotheses we have certain clues to demonstrate that when the adipocytes are treated with dexamethasone, which is known to induce insulin resistance, Atp10c expression increases (Fig. 5), suggesting that an increased Atp10c expression might result in a loss of insulin sensitivity. If this is proved correct then conversely, a decrease in Atp10c expression should decrease insulin resistance thereby improve insulin sensitivity? This is supported by the expression patterns observed in presence of TZDs (Fig. 4).

In all types of obesity and type 2 diabetes, the major abnormality lies in the glucose uptake system. In peripheral tissues, insulin-stimulated glucose uptake is dependent upon the translocation of insulin-responsive glucose transporter GLUT4 from intracellular storage compartments, the trans Golgi network to the plasma membrane. Membrane trafficking of GLUT4 is a complex phenomenon involving three major steps - insulin signaling to GLUT4 vesicles, trafficking of GLUT4 vesicles to the plasma membrane and finally docking and fusion of GLUT4 vesicles with the plasma membrane $[43,44]$. In view of our data, it is conceivable that the translocase activities of ATP10C protein mediate GLUT4 translocation at any one of these steps. Changes in Atp10c gene expression might affect the surface properties, membrane curvature, vesicle formation, fusion, or bilayer thickness/fluidity of the plasma membrane thereby affecting the trafficking and/or biogenesis of glucose transporters. Focused, hypothesis-driven experiments to address these questions can now be initiated in our cell systems.

\section{ACKNOWLEDGEMENTS}

The authors thank Dr. David West and Dr. Francine Gregoire for helpful discussions during the preparation of this manuscript. This work was supported by grants from the Center of Excellence and Comparative and Experimental Medicine programs of the University of Tennessee.

\section{REFERENCES}

[1] Graham T. Flippases and vesicle-mediated protein transport. Trends Cell Biol 2004; 14: 670-5.

[2] Paulusma CC, Oude ERPJ. The type 4 subfamily of P-type ATPases, putative aminophospholipid translocases with a role in human disease. Biochim Biophys Acta 2005; 1741: 11-24.

[3] Daleke DL. Phospholipid flippases. J Biol Chem 2007; 282: 821-5.

[4] Pomorski T, Holthuis JCM, Herrmann A, Meer G. Tracking down lipid flippases and their biological functions. J Cell Sci 2004; 117 : 805-13.

[5] Paulusma CC, Oude ERPJ. Diseases of intramembranous lipid transport. FEBS Lett 2006; 580: 5500-9.

[6] Bull LN, van Eijk MJ, Pawlikowska L, et al. A gene encoding a Ptype ATPase mutated in two forms of hereditary cholestasis. Nat Genet 1998; 18: 219-24.

[7] Paulusma CC, Groen A, Kunne C, et al. Atp8b1 deficiency in mice reduces resistance of the canalicular membrane to hydrophobic bile salts and impairs bile salt transport. Hepatology 2006; 44: 195-204.

[8] Flamant S, Pescher P, Lemercier B, et al. Characterization of a putative type IV aminophospholipid transporter P-type ATPase. Mamm Genome 2003; 14: 21-30.

[9] Dhar MS, Sommardahl CS, Kirkland T, et al. Mice heterozygous for Atp10c, a putative amphipath, represent a novel model of obesity and type 2 diabetes. J Nutrition 2004; 134: 799-805.

[10] Dhar MS, Yuan JS, Elliott SB, et al. A type IV P-type ATPase affects insulin-mediated glucose uptake in adipose tissue and skeletal muscle in mice. J Nutr Biochem 2006; 17: 811-20.

[11] Dhar M, Webb LS, Smith L, et al. A novel ATPase on mouse chromosome 7 is a candidate gene for increased body fat. Physiol Genomics 2004; 4: 93-100.

[12] Dhar MS, Hauser LJ, Nicholls RD, et al. Physical mapping of the pink-eyed dilution complex in mouse chromosome 7 shows that Atp $10 c$ is the only transcript between Gabrb3 and Ube3a. DNA Seq 2004; 15: 306-9.

[13] Nicholls RD, Knepper JL. Genome organization, function and imprinting in Prader-Willi and Angelman syndromes. Ann Rev Genomics Hum Genet 2001; 2: 153-75.

[14] Lossie AC, Whitney MM, Amidon D, et al. Distinct phenotypes distinguish the molecular classes of Angelman syndrome. J Med Genet 2001; 38: 834-45.

[15] Nicholls RD, Stefan M, Ji H, et al. Mouse models for Prader-Willi and Angelman syndromes offer insights into novel obesity mechanisms. In: Medeiros-Neto G, Halpern A, Bouchard C, Eds. Progress in Obesity Research. Montrouge, France: John Libbey Eurotext Ltd. 2003; 9: pp. 313-19. 
[16] Green H, Meuth M. An established pre-adipose cell line and its differentiation in culture. Cell 1974; 3: 127-33.

[17] Dhar MS, Johnson DK. A microsatellite map of the pink-eyed dilution ( $p$ ) deletion complex in mouse chromosome 7. Mamm Genome 1997; 8: 143-45.

[18] Rodbell M. Metabolism of isolated fat cells. I. Effects of hormones on glucose metabolism and lipolysis. J Biol Chem 1964; 239: 37580 .

[19] Koopman R, Schaart G, Hesselink MK. Optimization of Oil Red O staining permits combination with immunofluorescence and automated quantification of lipids. Histochem Cell Biol 2001; 116: 63-8.

[20] Ailhaud G, Grimaldi P, Negrel R. Cellular and molecular aspects of adipose tissue development. Annu Rev Nutr 1992; 212: 207-33.

[21] Shahparaki A, Grunder L, Sorisky A. Comparison of human abdominal subcutaneous versus omental preadipocyte differentiation in primary culture. Metabolism 2002; 51: 1211-15.

[22] Rosen ED, Walkey CJ, Puigserver P, et al. Transcriptional regulation of adipogenesis. Genes Dev 2000; 14: 1293-307.

[23] Sadowski HB, Wheeler TT, Young DA. Gene expression during 3T3-L1 adipocyte differentiation. J Biol Chem 1992; 266: 4722-31.

[24] Spiegelman BM, Frank M, Green H. Molecular cloning of mRNA from 3T3 adipocytes. Regulation of mRNA content for glycerophosphate dehydrogenase and other differentiation-dependent proteins during adipocyte development. J Biol Chem 1983; 258: 10083-89.

[25] Farmer SR. Regulation of PPARgamma activity during adipogenesis. Int J Obes (Lond) 2005; 29 (Suppl 1): S13-6.

[26] Yeh WC, Cao Z, Classon M, et al. Cascade regulation of terminal adipocyte differentiation by three members of the C/EBP family of leucine zipper proteins. Genes Dev 1995; 9: 168-81.

[27] Hammarstedt A, Andersson CX, Rotter SV, et al. The effect of PPARgamma ligands on the adipose tissue in insulin resistance. Prostaglandins Leukot Essent Fatty Acids 2005; 73: 65-75.

[28] Yamaguchi K, Lee SH, Eling TE, et al. A novel peroxisome proliferator-activated receptor gamma ligand, MCC-555, induces apoptosis via posttranscriptional regulation of NAG-1 in colorectal cancer cells. Mol Cancer Ther 2006; 5: 1352-61.
[29] Kershaw EE, Schupp M, Guan HP, et al. PPAR \{gamma\} regulates adipose triglyceride lipase in adipocytes in vitro and in vivo. Am J Physiol Endocrinol Metab 2007; 293(6): E1736-45.

[30] Mora S, Pessin JE. An adipocentric view of signaling and intracellular trafficking. Diabetes Metab Res Rev 2002; 18: 345-56.

[31] Bradley RL, Cleveland KA, Cheatham B. The adipocyte as a secretory organ: mechanisms of vesicle transport and secretory pathways. Recent Prog Horm Res 2001; 56: 329-58.

[32] Halleck MS, Pradhan D, Blackman C, et al. Multiple members of a third subfamily of P-type ATPases identified by genomic sequences and ESTs. Genome Res 1998; 8: 354-61.

[33] Halleck MS, Lawler JF, Blackshaw S, et al. Differential expression of putative transbilayer amphipath transporters. Physiol Genomics 1999; 1: 139-50.

[34] Williamson P, Schlegel RA. Transbilayer phospholipid movement and clearance of apoptotic cells. Biochim Biophys Acta 2002; 1585: 53-63.

[35] Axelsen KB, Palmgren, MG. Evolution of substrate specificities in the P-type ATPase superfamily. J Mol Evol 1998; 46: 84-101.

[36] Rosen ED, MacDougald OA. Adipocyte differentiation from the inside out. Nat Rev 2006; 7: 885-96.

[37] Ntambi JM, Kim YC. Adipocyte differentiation and gene expression. J Nutr 2000; 130: 3122S-3126S.

[38] Wang Y, Kim KA, Kim JH, et al. Pref-1, a preadipocyte secreted factor that inhibits adipogenesis. J Nutr 2006; 136: 2953-56.

[39] Sato M, Hiragun A, Mitsui H. Preadipocytes possess cellular retinoid binding proteins and their differentiation is inhibited by retinoids. Biochem Biophys Res Commun 1980; 95: 1839-45.

[40] Vaulont S, Kahn A. Transcriptional control of metabolic regulation genes by carbohydrates. FASEB J 1994; 8: 28-35.

[41] Desvergne B, Michalik L, Wahli W. Transcriptional regulation of metabolism. Physiol Rev 2006; 86: 465-514.

[42] Fasshauer M, Paschke R. Regulation of adipocytokines and insulin resistance. Diabetologia 2003; 46: 1594-1603.

[43] Kanzaki M. Insulin receptor signals regulating GLUT4 translocation and actin dynamics. Endocr J 2006; 53(3): 267-93.

[44] Watson RT, Pessin JE. GLUT4 translocation: the last 200 nanometers. Cell Signal 2007; 19(11): 2209-17.

Received: January 09, 2009

Revised: February 01, 2009

Accepted: February 02, 2009

(C) Peretich et al.; Licensee Bentham Open.

This is an open access article licensed under the terms of the Creative Commons Attribution Non-Commercial License (http://creativecommons.org/licenses/by$\mathrm{nc} / 3.0 /$ ), which permits unrestricted, non-commercial use, distribution and reproduction in any medium, provided the work is properly cited. 